\title{
Distribución y abundancia de la comunidad de peces en la porción litoral de la Reserva de la Biósfera Los Petenes, Campeche, México
}

\author{
Sandra Muñoz-Rojas ${ }^{1}$, Luis Amado Ayala-Pérez ${ }^{1}$, Atahualpa Sosa-López ${ }^{2}$ \&
}

Guillermo Jorge Villalobos-Zapata ${ }^{2}$

1. Universidad Autónoma Metropolitana Xochimilco, Departamento El Hombre y su Ambiente, Calz. Del Hueso 1100 Col. Villaquietud, Coyoacan 04960, México D.F.; samuro_08@yahoo.com.mx, luayala@correo.xoc.uam.mx

2. Universidad Autónoma de Campeche, Instituto de Ecología Pesquerías y Oceanografía del Golfo de México, Av. Agustín Melgar s/n entre Juan de la Barrera y Calle 20 Col. Buenavista 24030 Campeche, Campeche; atahsosa@ uacam.mx, gjvillal@uacam.mx

Recibido 21-XI-2011. C Corregido 10-VIII-2012. Aceptado 19-IX-2012.

\begin{abstract}
Distribution and abundance of fish community in the littoral area of "Los Petenes" Biosphere Reserve, Campeche, Mexico. "Los Petenes" Biosphere Reserve (RBLP) is a critical habitat for many aquatic and terrestrial species. It has the biggest and better conserved seagrass beds, and it represents an important habitat for food, protection and breeding of aquatic organisms, and a temporal refuge for migratory species. The objective of this study was to describe the ichthyofauna diversity in the littoral coastal area of the RBLP, to identify the ecological dominant species, and to analyze the abundance of the fish community and its temporal and spatial changes, and their relationship with some environmental variables. Monthly fish samples were obtained with the aid of trawl nets, from 24 samplings sites distributed along the reserve, between May 2009 and April 2010. The trawl net was operated 288 times and 21795 individuals with $279.5 \mathrm{~kg}$ of weight were collected. A total of 46 fish species grouped in 34 genera and 23 families were identified. In a spatial scale, the abundance showed the next ranges: $0.018-0.094 \mathrm{ind} . / \mathrm{m}^{2} ; 0.249-1.072 \mathrm{~g} / \mathrm{m}^{2}$ and $9.75-19.32 \mathrm{~g} / \mathrm{ind}$.; the diversity indexes obtained were: $H^{\prime} n=1.46-2.15, J^{\prime}=0.45-0.71$ and $D^{\prime}=2.08-3.92$. In a temporal scale, the abundance and diversity ranged between: $0.026-0.066 \mathrm{ind} . / \mathrm{m}^{2} ; 0.342-0.764 \mathrm{~g} / \mathrm{m}^{2}$ and $6.49-22.98 \mathrm{~g} / \mathrm{ind}$.; H'n=1.76-2.08; $\mathrm{J}=0.52-0.64$ and $\mathrm{D}=3.07-4.18$. Eleven dominant species were identified with a representation of the $94.39 \%$ in number of individuals, and $89.66 \%$ in weight of the total catch. From the total, eight species had economic or commercial importance, especially Lagodon rhomboides and Haemulon plumierii. The cluster analyses identified four fish associations; these results are discussed in order to identify relationships between habitat-species. Finally, the canonical correspondence analysis evidenced an association between $H$. plumierii with salinity and dissolved solids. The RBLP has high habitat diversity and its fish community has developed strategies to use all the spatial and temporal conditions and to satisfy the needs of their life cycles. Rev. Biol. Trop. 61 (1): 213-227. Epub 2013 March 01.
\end{abstract}

Key words: abundance, diversity, fish community, Los Petenes, Campeche.

La Reserva de la Biósfera Los Petenes (RBLP) se localiza en la zona costera Norte del Estado de Campeche, conforma una unidad biogeográfica única de gran importancia biológica, ecológica y científica derivada de su alta diversidad de flora y fauna así como de ecosistemas (Corbalá et al. 2007).

Los Petenes son biotopos que sólo se localizan en la Península de Yucatán, en Cuba y en la Península de la Florida. La RBLP además ha sido declarada sitio RAMSAR en el 2004 reconociéndose su valor como humedal internacional (Villalobos-Zapata 2004), y su relevancia ecológica se centra en ser hábitat crítico para muchas especies tanto acuáticas como terrestres. Cuenta con la mayor y mejor conservada superficie de pastos marinos que son utilizados como áreas de alimentación, 
protección y crianza de organismos acuáticos y zona de refugio temporal para especies migratorias (CONANP 2006).

Pese a su evidente importancia ecológica y a la intensa actividad pesquera que se desarrolla en la porción marina de la RBLP, la información disponible sobre la diversidad de hábitat y de fauna acuática es escasa, y algunos trabajos se han enfocado principalmente hacia la descripción de la vegetación y fauna terrestre (Rico-Gray 1982, Durán 1995, Mas \& Correa 2000).

Algunos trabajos de referencia comparativa sobre la ictiofauna corresponden a ecosistemas adyacentes tal como lo reportado por Vega-Cendejas (2004) y Villalobos-Zapata (2004) para la Reserva de la Biósfera Ría Celestún y Petenes, asimismo Méndez-Cabrera \& Montiel (2007) describen el aprovechamiento de flora y fauna silvestre en las comunidades "La isla" y "El remate", y Torres-Castro et al. (2008) comparan los petenes de Hampolol y el Remate, en términos de su calidad ambiental y fauna de peces.

A pesar de que los peces constituyen la macrofauna acuática más abundante y que además funcionan como reguladores energéticos a lo largo de la cadena trófica y como recicladores de nutrientes entre los diversos componentes del ecosistema (Ramos-Miranda et al. 2009), los estudios sistemáticos de este componente, son escasos y proporcionan información parcial. Por lo que este estudio tiene como objetivos describir la abundancia y diversidad de la ictiofauna, analizar su estructura espacial y temporal y su relación con algunas variables ambientales e identificar a las especies con dominio ecológico.

\section{MATERIALES Y MÉTODOS}

El área de estudio se localiza al Sureste del Golfo de México en el estado de Campeche (Fig. 1), la RBLP ocupa una extensión de 282 857 ha entre los $20^{\circ} 51^{\prime} 30^{\prime \prime}-19^{\circ} 49^{\prime} 00^{\prime \prime} \mathrm{N}$ y los $90^{\circ} 45^{\prime} 15^{\prime}$ - 90²0’00”'W. Sus límites son: al Norte la Reserva de la Biósfera Ría Celestún y el Golfo de México; al Oriente colinda con los municipios de Tenabo, Hecelchakán y Calkiní, y al Sur con la ciudad de San Francisco de Campeche. La Reserva se localiza en la región hidrológica 32 que pertenece a la cuenca hidrológica Yucatán Norte. Como consecuencia de la naturaleza kárstica del terreno y su poco relieve, los escurrimientos superficiales son prácticamente nulos y los ríos subterráneos desembocan en la plataforma continental como ojos de agua o manantiales (Torres-Castro et al. 2008).

Según la CONANP (2006) el clima predominante en la zona centro-sur de la Reserva es Aw (cálido subhúmedo con lluvias en verano), mientras que en su extremo norte es de tipo BS'h'w (semiseco y seco cálido). La temperatura y precipitación media anual varía de $27.8^{\circ} \mathrm{C}$ y $725.5 \mathrm{~mm}$ en el Norte (con un gradiente entre los $700-800 \mathrm{~mm})$, a $26.4^{\circ} \mathrm{C}$ y $1049.7 \mathrm{~mm}$ (con un gradiente de $800-1100 \mathrm{~mm}$

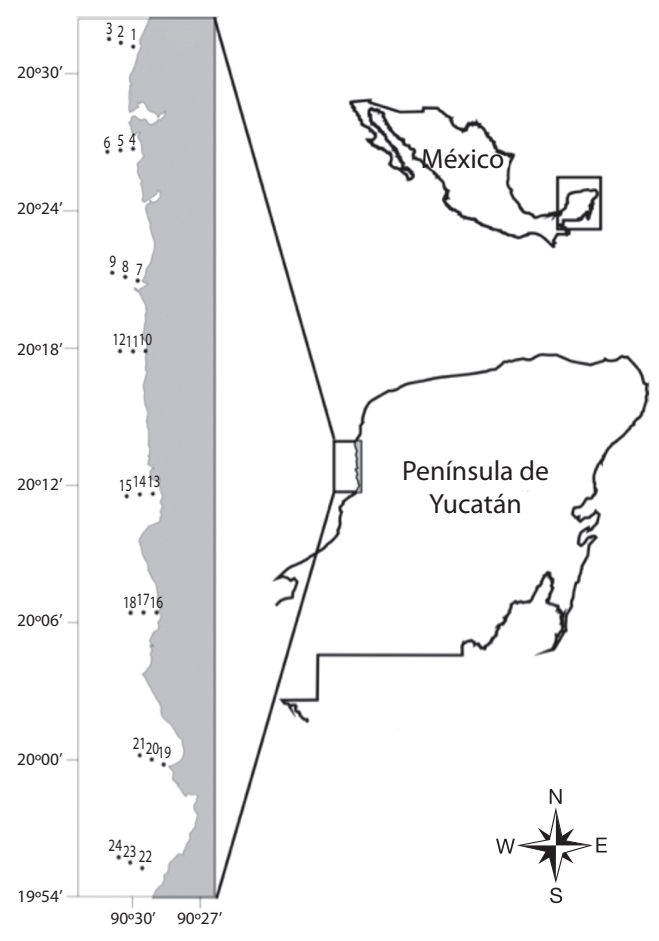

Fig. 1. Sitios de muestreo en la Reserva de la Biósfera Los Petenes.

Fig. 1. Sites in Los Petenes Biosphere Reserve. 
anuales en el Sur). Presenta un breve período de sequía durante lluvias (sequía intraestival o canícula). A pesar de que Lara-Lara et al. (2008) describen la presencia de tres épocas climáticas para todo el Golfo de México, en la zona de estudio la variación ambiental específica se explica fundamentalmente con dos épocas climáticas, la época de secas que va de noviembre a abril y la época de lluvias de mayo a octubre (CONANP 2006).

De mayo del 2009 a abril del 2010 se tomaron muestras mensualmente en 24 sitios distribuidos en el área de estudio. Los sitios de muestreo fueron ubicados considerando algunos criterios geomorfológicos representativos, tales como bahías, ensenadas, efluentes costeros, islas costeras, vegetación sumergida y circundante, de manera que se tuviese representado cada ambiente. En cada sitio se realizaron mediciones de parámetros ambientales (temperatura, salinidad, oxígeno disuelto y $\mathrm{pH})$ del agua en dos niveles de profundidad (superficie y fondo) empleando una sonda multiparamétrica (Hydrolab DS5). Las muestras biológicas se obtuvieron con una red de arrastre camaronera de prueba de $5 \mathrm{~m}$ de largo, $2.5 \mathrm{~m}$ de abertura de trabajo y $19 \mathrm{~mm}$ de luz de malla, operada por $12 \mathrm{~min}$ a bordo de una lancha con motor fuera de borda a una velocidad de 2.5 nudos. Los organismos capturados se almacenaron en bolsas de plástico etiquetadas y se conservaron en hielo.

En el laboratorio los organismos fueron lavados e identificados utilizando literatura especializada (Fischer 1978, Cervigón et al. 1992, Castro-Aguirre 1999) y de manera individual se registró la talla total $(\mathrm{cm})$ y peso total (g) con una balanza digital de $2160 \mathrm{~g}$ y $0.1 \mathrm{~g}$ de precisión.

Se presenta en forma gráfica las variaciones ambientales del ecosistema (salinidad, temperatura, $\mathrm{pH}$ y oxígeno disuelto) a nivel espacial como temporal. Los resultados se integraron en forma de gráficos de caja donde destacan los valores de la mediana, interquartiles primero y tercero y los valores mínimo y máximo, con apoyo del programa estadístico SYSTAT ver. 10.2 (Systat Software Inc. 2002).
Para determinar la abundancia relativa se estimó el área barrida por el arte de pesca considerando el producto de la abertura de trabajo por la velocidad y el tiempo de arrastre. La abundancia se evaluó en términos de densidad (ind. $\left./ \mathrm{m}^{2}\right)$, biomasa $\left(\mathrm{g} / \mathrm{m}^{2}\right)$ y peso promedio $(\mathrm{g} /$ ind.) y se analizó en escalas espacial y temporal. La diversidad de la comunidad de peces se determinó con ayuda de las expresiones matemáticas propuestas por Shanon \& Weaver (1963) $\left(H^{\prime} n\right)$, Margalef (1969) (D) y Pielou $(1966)\left(J^{\prime}\right)$ acorde a las siguientes expresiones:

$$
\begin{gathered}
\mathrm{H}^{\prime} \mathrm{n}=-\sum_{\mathrm{i}=1}^{n i} \mathrm{ni} / \mathrm{N} * \ln (\mathrm{ni} / \mathrm{N}) \\
\mathrm{D}^{\prime}=\mathrm{S}-1 / \ln (\mathrm{N}) \\
\mathrm{J}^{\prime}=\mathrm{H}^{\prime} / \ln (\mathrm{S})
\end{gathered}
$$

Donde: H'n=índice de diversidad, D`=índice de riqueza de especies, $\mathrm{J}_{\rangle}=$índice de equidad, ni=número de individuos de la especie $i, N=$ número total de individuos de todas las especies, $\mathrm{S}=$ número de especies, $\ln =$ logaritmo natural.

Para la determinación de las especies dominantes se consideró la abundancia numérica, abundancia en peso y frecuencia de aparición de acuerdo al índice de importancia relativa modificado por Koranteng (2001) y que se define como:

$$
\mathrm{IRI}=\% \mathrm{~W} * 0 \mathrm{~N} * 0 \mathrm{~F}
$$

Donde: $\% \mathrm{~W}=$ Porcentaje de la contribución en peso de la especie a la captura total; $\% \mathrm{~N}=$ Porcentaje de la contribución en número de la especie a la captura total; $\% \mathrm{~F}=$ Porcentaje de la frecuencia de aparición de la especie en relación con el número total de estaciones. Las especies con valores IRI $\geq 20$ se consideran como especies dominantes, si el IRI $<20 \mathrm{y} \geq 1$ se consideran como de importancia media y si el IRI $<1$, se consideran como de baja importancia.

Para la determinación de los conjuntos ictiofaunísticos dominantes se efectuó un análisis de agrupamiento (cluster) empleando una 
matriz de número de individuos por especie, estación y mes, aplicando el método de ligamiento Ward con distancia Gamma, con apoyo del programa estadístico SYSTAT versión 10.2 (Systat Software Inc. 2002).

El análisis de la asociación entre las especies dominantes con los parámetros ambientales se realizó por medio de un Análisis Canónico de Correspondencias (ACC) para lo cual se utilizaron las matrices de número de individuos por especie, sitio de recolecta y valores de los parámetros ambientales por sitio de muestreo, empleando el paquete computacional CANOCO 4.5 (Braak \& Šmilauer 2002).

\section{RESULTADOS}

Un resumen de los valores de temperatura, salinidad, oxígeno disuelto y $\mathrm{pH}$ de superficie y fondo durante el periodo de muestreo se presentan en el cuadro 1 y se acompañan de la estadística básica. En las figuras 2 y 3 se representa la variación espacial y temporal de los parámetros ambientales. Los valores de superficie y fondo de los parámetros ambientales fueron sometidos a un análisis estadístico de comparación de medias y se encontró que no existen diferencias significativas $(\mathrm{p}>0.05)$, por lo que para la escala espacial se reportan

CUADRO 1

Estadística descriptiva de los parámetros ambientales en la Reserva de la Biósfera Los Petenes agrupada por época climática

TABLE 1

Descriptive statistics of environmental parameters in Los Petenes Biosphere Reserve grouped by climatic season

\begin{tabular}{|c|c|c|c|c|}
\hline \multirow{2}{*}{ Temperatura } & \multicolumn{2}{|c|}{ Secas } & \multicolumn{2}{|c|}{ Lluvias } \\
\hline & Superficie & Fondo & Superficie & Fondo \\
\hline Mínimo & 17.3 & 17.3 & 28.2 & 28.2 \\
\hline Máximo & 27.9 & 27.9 & 32.4 & 32.9 \\
\hline Promedio & 23.9 & 23.9 & 30.1 & 30.1 \\
\hline Desv. estándar & 2.7 & 2.7 & 1.04 & 1.0 \\
\hline Varianza & 7.7 & 7.4 & 1.08 & 1.0 \\
\hline \multicolumn{5}{|l|}{ Salinidad } \\
\hline Mínimo & 25.9 & 27.5 & 33.6 & 34.4 \\
\hline Máximo & 42.5 & 42.0 & 47.9 & 43.5 \\
\hline Promedio & 35.7 & 35.9 & 38.1 & 38.1 \\
\hline Desv. estándar & 3.7 & 3.5 & 2.3 & 2.0 \\
\hline Varianza & 14.1 & 12.8 & 5.7 & 4.1 \\
\hline \multicolumn{5}{|l|}{ Oxígeno Disuelto } \\
\hline Mínimo & 2.1 & 2.1 & 3.1 & 2.2 \\
\hline Máximo & 10.7 & 9.0 & 11.8 & 11.9 \\
\hline Promedio & 6.4 & 6.3 & 6.5 & 6.6 \\
\hline Desv. estándar & 1.4 & 1.4 & 1.9 & 1.9 \\
\hline Varianza & 2.0 & 2.1 & 3.6 & 3.9 \\
\hline \multicolumn{5}{|l|}{ pH } \\
\hline Mínimo & 6.9 & 6.9 & 7.8 & 7.8 \\
\hline Máximo & 9.5 & 9.4 & 9.1 & 9.1 \\
\hline Promedio & 8.1 & 8.1 & 8.4 & 8.4 \\
\hline Desv. estándar & 0.8 & 0.8 & 0.3 & 0.3 \\
\hline Varianza & 0.7 & 0.7 & 0.0 & 0.1 \\
\hline
\end{tabular}



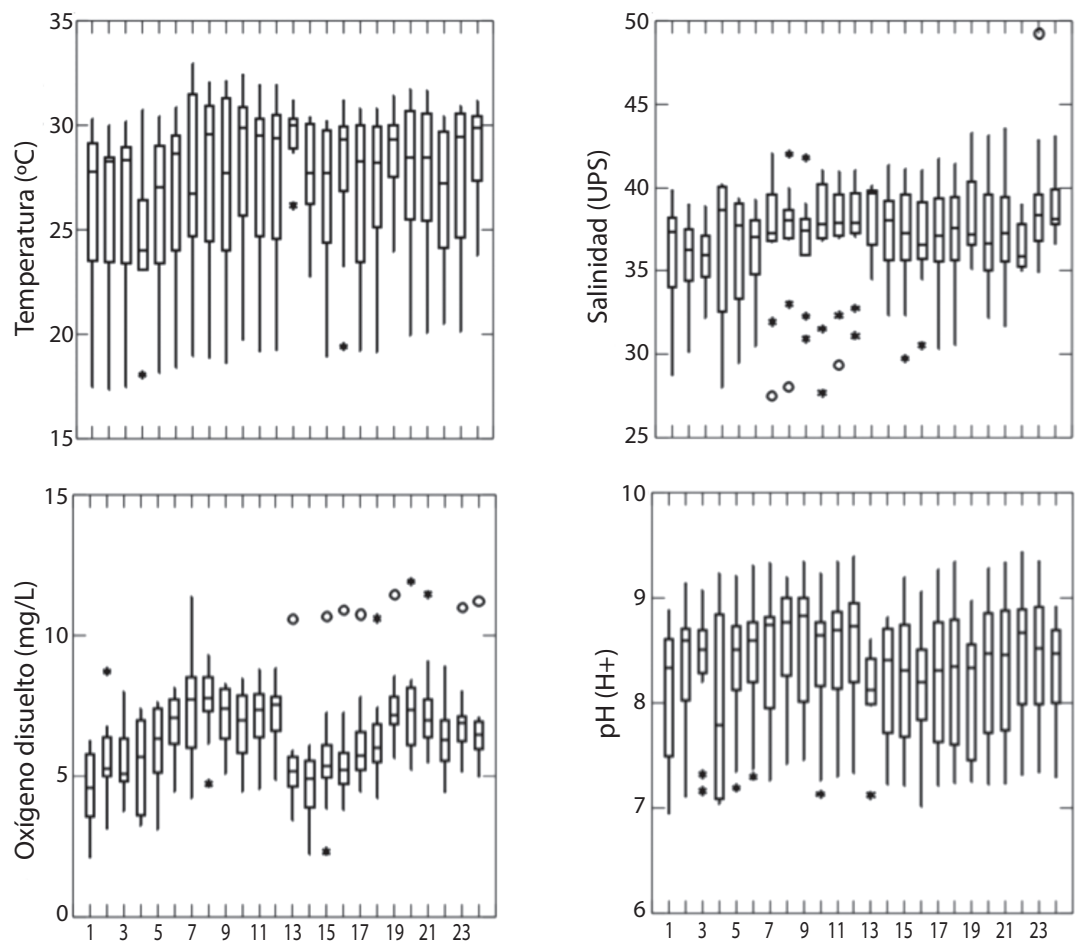

Fig. 2. Comportamiento espacial de los parámetros ambientales de fondo en la Reserva de la Biósfera Los Petenes (*=valores atípico leves $\mathrm{o}=$ valores atípicos extremos).

Fig. 2. Spatial behavior of environmental parameters of bottom in Los Petenes Biosphere Reserve $(*=$ mild atypical values $\mathrm{o}=$ extreme outliers).

solo los valores de fondo. Para todos los sitios de recolecta, el promedio de la temperatura fue de $27.1^{\circ} \mathrm{C}$; la salinidad osciló entre 27.5 UPS y 43.5 UPS. El pH mostró una variación entre 8.6 y 9.5 , y finalmente, el oxígeno disuelto presentó un intervalo de variación de 7.4 y $11.9 \mathrm{mg} / \mathrm{L}$. La variación de la profundidad en función de la ubicación de los sitios de muestreo se representa en la figura 4.

Se realizaron 288 arrastres, capturándose un total de 21795 organismos con un peso total de $279.5 \mathrm{~kg}$. Se identificaron 46 especies de peces agrupadas en 34 géneros y 23 familias. La abundancia de la comunidad de peces mostró los siguientes intervalos de variación en escala espacial: $0.018-0.094 \mathrm{ind} . / \mathrm{m}^{2} ; 0.249$ $1.072 \mathrm{~g} / \mathrm{m}^{2}$ y $9.75-19.32 \mathrm{~g} /$ ind. La diversidad de la comunidad se representó por el índice de Shanon con valores entre 1.46-2.15bits/ind. El valor de los índices de equidad y riqueza de especies presentó una variación entre 0.45-0.71 y 2.08-3.92, respectivamente. En escala temporal, los intervalos de variación de los parámetros de abundancia y diversidad encontrados fueron los siguientes: $0.026-0.066 \mathrm{ind} . / \mathrm{m}^{2}$; $0.342-0.764 \mathrm{~g} / \mathrm{m}^{2}$ y $6.49-22.98 \mathrm{~g} /$ ind. H'n=1.762.08bits/ind.; $\mathrm{J}=0.52-0.64$ y $\mathrm{D}=3.07-4.18$. El comportamiento espacial y temporal de estos parámetros ecológicos se presentan en las figuras 5 y 6 .

Se identificaron once especies dominantes de las cuales ocho tienen importancia económica o comercial, juntas representaron el $94.39 \%$ en número de individuos y el $89.66 \%$ en peso de la captura total (Cuadro 2). En la figura $7 \mathrm{se}$ presenta el resultado del análisis clúster donde se observa la formación de cuatro grupos a una distancia de 1.0. El primer grupo estuvo 

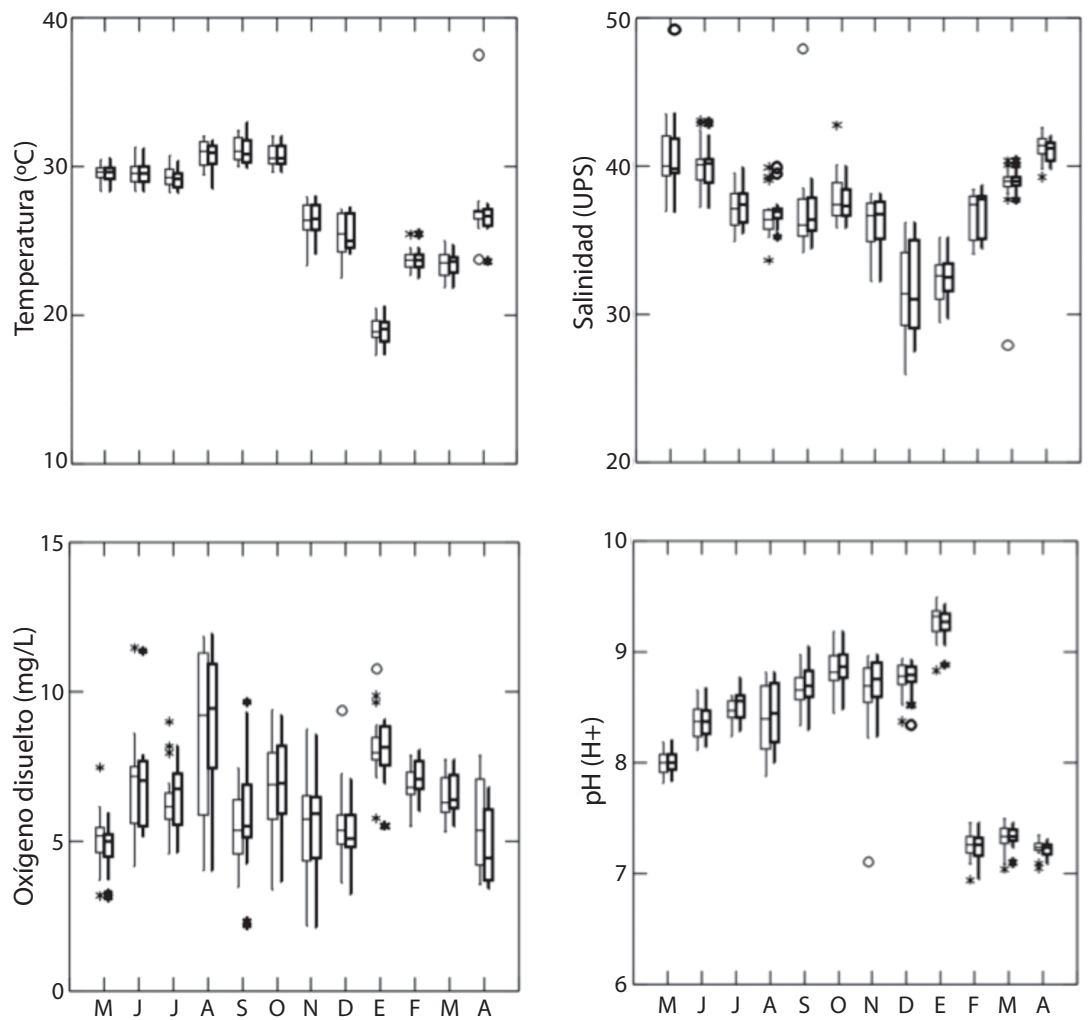

Fig. 3. Comportamiento temporal de los parámetros ambientales en dos niveles de profundidad en la Reserva de la Biósfera Los Petenes $(*=$ valores atípico leves $\mathrm{o}=$ valores atípicos extremos).

Fig. 3. Temporal behavior of environmental parameters in two levels of depth in Los Petenes Biosphere Reserve $(*=$ mild atypical values $\mathrm{o}=$ extreme outliers).

CUADRO 2

Especies dominantes de la Reserva de la Biósfera Los Petenes

TABLE 2

Dominant species of Los Petenes Biosphere Reserve

\begin{tabular}{lcccccc}
\multicolumn{1}{c}{ Especie } & No. ind. & $\begin{array}{c}\text { No. ind. } \\
(\%)\end{array}$ & $\begin{array}{c}\text { Peso total } \\
(\mathrm{kg})\end{array}$ & $\begin{array}{c}\text { Peso } \\
(\%)\end{array}$ & $\begin{array}{c}\text { Frecuencia } \\
(\%)\end{array}$ & $\begin{array}{c}\text { Índice de } \\
\text { importancia relativa }\end{array}$ \\
Lagodon rhomboides & 8673 & 39.1 & 96.4 & 33.7 & 90.9 & 3068.2 \\
Haemulon plumierii & 5346 & 24.5 & 56.0 & 20.0 & 94.1 & 1885.1 \\
Archosargus rhomboidalis & 1181 & 5.1 & 28.7 & 8.5 & 64.9 & 553.2 \\
Orthopristis chrysoptera & 1385 & 6.3 & 18.1 & 6.4 & 65.2 & 424.1 \\
Opsanus beta & 399 & 1.8 & 18.2 & 6.5 & 42.3 & 275.3 \\
Eucinostomus gula & 2266 & 10.3 & 7.6 & 2.7 & 81.9 & 223.9 \\
Acanthostracion quadricornis & 232 & 1.0 & 10.2 & 3.6 & 48.6 & 177.1 \\
Stephanolepis hispida & 479 & 2.1 & 5.3 & 1.9 & 52.0 & 99.7 \\
Calamus penna & 432 & 1.9 & 5.9 & 2.1 & 38.1 & 81.0 \\
Nicholsina usta & 276 & 1.2 & 5.7 & 2.0 & 30.9 & 62.8 \\
Synodus foetens & 104 & 0.4 & 5.6 & 1.9 & 27.4 & 52.3
\end{tabular}




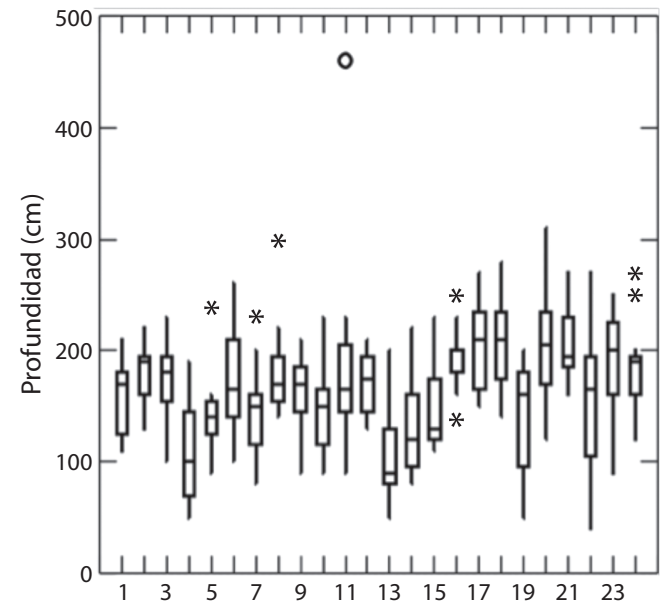

Fig. 4. Variación de la profundidad en los sitios de muestreo de la Reserva de la Biósfera los Petenes (*=valores atípico leves $\mathrm{o}=$ valores atípicos extremos).

Fig. 4. Depth variation of the sampling sites in Los Petenes Biosphere Reserve $(*=$ mild atypical values $\mathrm{o}=$ extreme outliers).
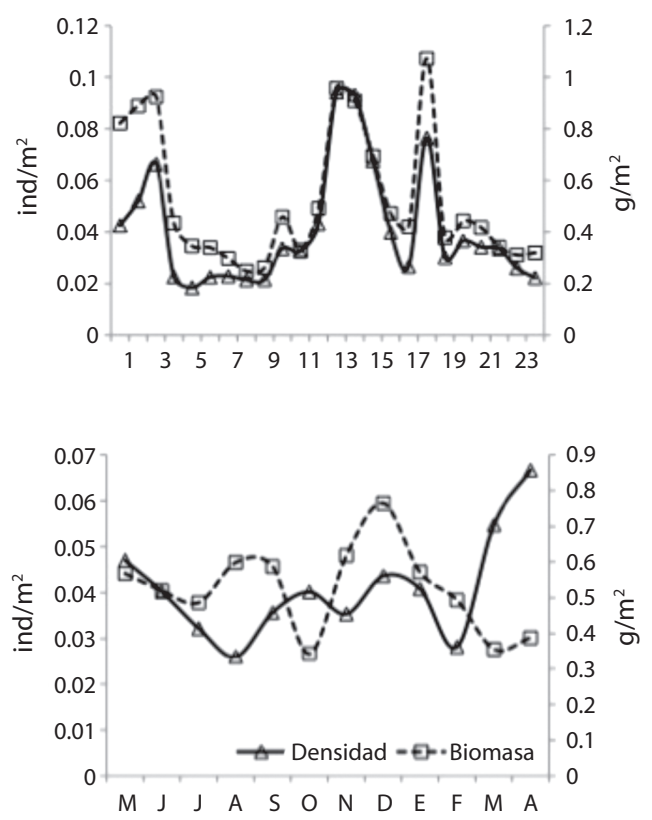

integrado por Acanthostracion quadricornis (ACQU) y Calamus penna (CAPE), el segundo grupo lo conforman Synodus foetens (SYFO), Nicholsina usta (NIUS) y Stephanolepis hispidus (STHI), el tercero lo constituyeron Haemulon plumierii (HAPL), Eucinostomus gula (EUGU), Orthopristis chrysoptera (ORCH) y Lagodon rhomboides (LARH) y finalmente el cuarto grupo estuvo integrado por Archosargus rhomboidalis (ARRH) y Opsanus beta (OPBE).

La figura 8 muestra la relación entre los parámetros ambientales de temperatura (TEMP), salinidad (SALI), $\mathrm{pH}$, oxígeno disuelto (OXIG) y sólidos disueltos (SOLI) y la abundancia de las especies de peces dominantes. Destacan las asociaciones entre salinidad y sólidos disueltos con H. plumierii (HAPL), temperatura y $\mathrm{pH}$ con $N$. usta (NIUS), E. gula (EUGU) y O. chrysoptera (ORCH) y oxígeno disuelto con $C$. penna (CAPE) y $S$. foetens (SYFO). Finalmente, O. beta (OPBE)
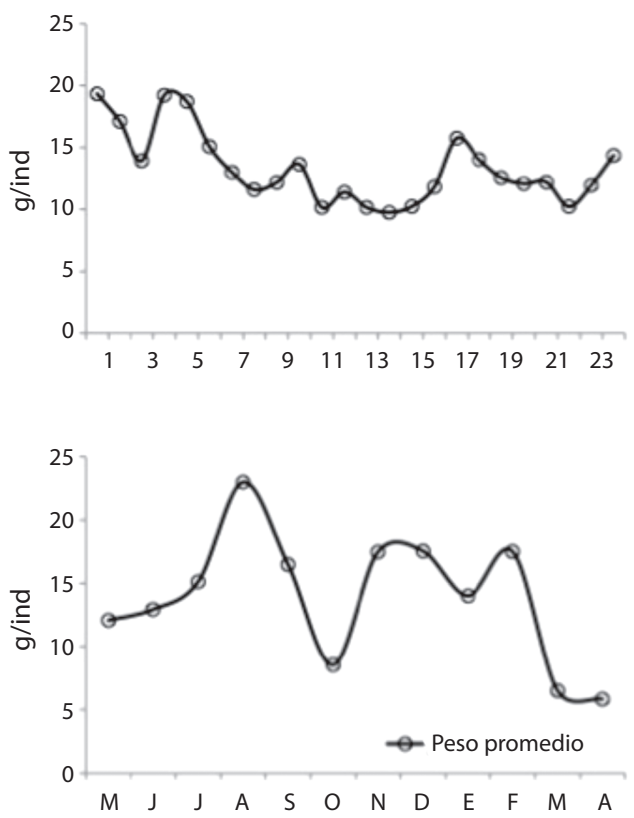

Fig. 5. Comportamiento espacial y temporal de la abundancia de la comunidad de peces en la Reserva de la Biósfera Los Petenes.

Fig. 5. Spatial and temporal behavior of the abundance of fish community in Los Petenes Biosphere Reserve. 

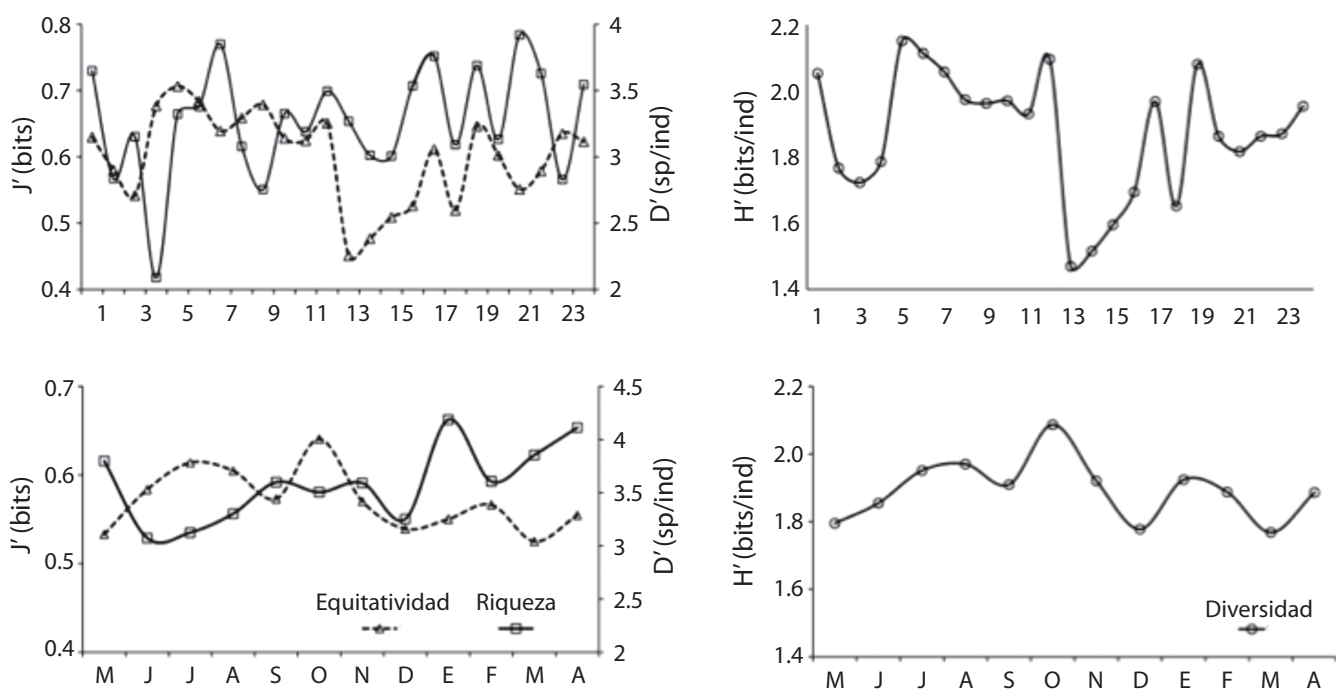

Fig. 6. Comportamiento espacial y temporal de los parámetros ecológicos de la comunidad de peces en la Reserva de la Biósfera Los Petenes.

Fig. 6. Spatial and temporal behavior of the ecological parameters of fish community in Los Petenes Biosphere Reserve.

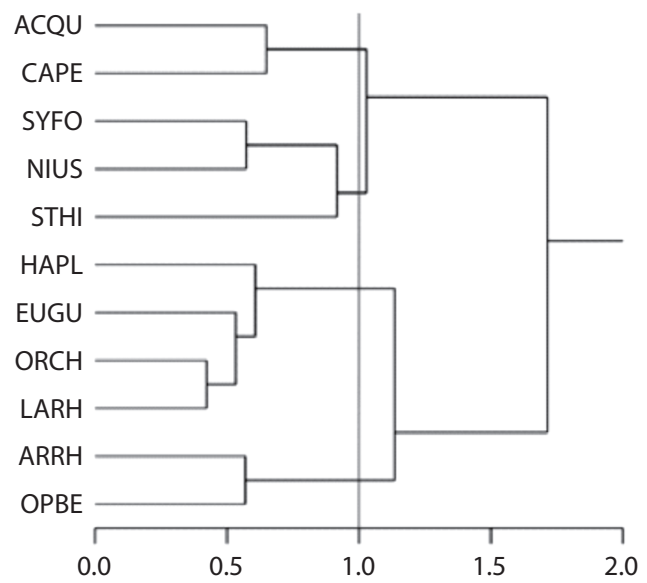

Fig. 7. Distribución de la abundancia de los peces dominantes de la Reserva de la Biósfera Los Petenes.

Fig. 7. Abundance distribution of dominant species of Los Petenes Biosphere Reserve.

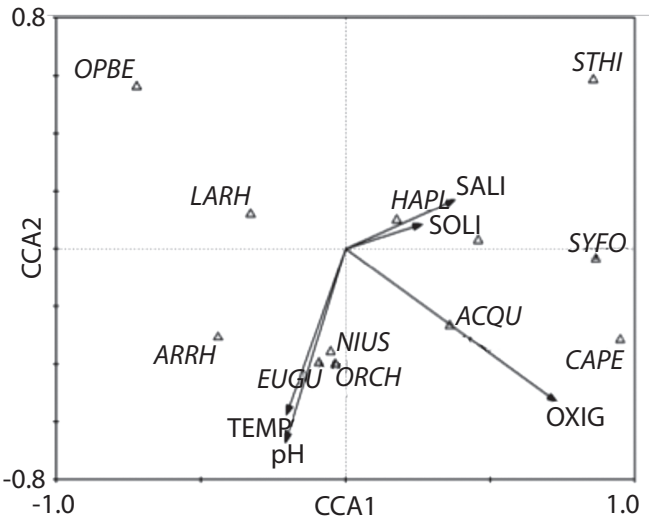

Fig. 8. Análisis de Correspondencia Canónica de la densidad de las especies dominantes y su relación con las variables ambientales en la Reserva de la Biósfera Los Petenes.

Fig. 8. Canonical Correspondence Analysis of the density of dominant species and its relation to environmental variables of Los Petenes Biosphere Reserve. 
y L. rhomboides (LARH) se identifican como especies de gran tolerancia que no tienen una asociación evidente con los parámetros ambientales registrados.

\section{DISCUSIÓN}

La reserva de la Biósfera Los Petenes pertenece a la región costera nerítica Campechano-Yucateca Interior de la plataforma Sur del Golfo de México, acorde a la clasificación de eco regiones marinas de México. La variabilidad ambiental en esta región está condicionada principalmente por procesos oceanográficos como el sistema de corrientes, procesos climático-meteorológicos como la precipitación y el volumen de descarga fluvial, y por una condición geográfica como la extensión de la plataforma continental y la ubicación latitudinal (Lara-Lara et al. 2008).

En una visión global del comportamiento ambiental del Golfo de México, el periodo de estiaje se identifica de febrero a mayo, el de lluvias de verano de junio a octubre con presencia de depresiones tropicales, y el periodo de frentes fríos anticiclónicos (nortes) que se presenta de octubre a febrero (Wiseman \& Sturges 1999). Sin embargo, de manera particular para la costa Norte de Campeche y de acuerdo a los registros de diez años de temperatura ambiente y precipitación pluvial de las estaciones meteorológicas de Campeche, Campeche y Celestún, Yucatán, el comportamiento ambiental se resume en dos época climáticas: Secas (Noviembre a Abril) y Lluvias (Mayo a Octubre) lo cual coincide con lo reportado por CONANP (2006). Los efectos ambientales que tienen las tormentas invernales provocadas por los frentes fríos durante la época de nortes en otras regiones, como la Laguna de Términos (Yáñez-Arancibia \& Day 1982, Ayala-Pérez 2006), no son tan evidentes en la RBLP.

La circulación de agua en el Golfo de México está definida principalmente por la fuerte corriente proveniente del Mar Caribe que penetra por el Estrecho de Yucatán y forma la denominada Corriente del Lazo para salir hacia el Atlántico Norte por el Estrecho de la Florida
(Vukovich 1988, Vidal et al. 1994). Esta condición de circulación se suma a las características particulares del área de estudio donde dominan fondos de tipo kárstico, hay alta transparencia y parches de vegetación sumergida, la plataforma continental es extensa con una pendiente muy suave, de manera que en los sitios de muestreo la profundidad raramente supera los dos metros. La marea es diurna con una variación cercana a $\operatorname{los} 30 \mathrm{~cm}$ y se identifican afloramientos de agua dulce y esteros que acumulan escurrimientos de agua continental. Suárez-Morales \& RiveraArriaga (1998) y Cervantes (2007) describen parte de la geomorfología de la península de Yucatán enfatizando el origen y funcionamiento de cenotes, petenes y ríos subterráneos como elementos centrales en el balance hídrico de la región y ayudan a entender las variaciones en la salinidad y $\mathrm{pH}$ en la porción costera litoral.

El contraste en el comportamiento de la temperatura, salinidad, oxígeno disuelto y $\mathrm{pH}$ de la columna de agua entre las dos épocas climáticas se asocia principalmente a precipitación pluvial local y a la aportación continental de agua acumulada en toda la planicie costera. Es de destacar el comportamiento de la temperatura y la salinidad en la época de lluvias, que inician con valores altos que paulatinamente van descendiendo similar a lo reportado por Ayala-Pérez et al. (2003) y Bautista et al. (2005) como una respuesta con retraso a la precipitación pluvial que se acumuló en la planicie costera y que tarda en llegar a la costa.

El comportamiento del oxígeno disuelto en el agua se interpreta como una respuesta al efecto combinado de procesos físicos como la circulación litoral, la marea, los vientos y la temperatura, procesos biológicos como la productividad primaria y la oxidación de la materia orgánica, y procesos químicos como el intercambio gaseoso con la atmósfera. En este sentido el intervalo de variación registrado refleja un ambiente altamente productivo, muy dinámico y es acorde a lo reportado por Lohrenz et al. (1999) que describe cómo las áreas costeras afectadas por ríos pequeños y estuarios, presentan valores elevados de productividad. 
En el caso del $\mathrm{pH}$ que muestra una tendencia hacia la alcalinidad refleja el efecto de lavado de sedimentos carbonatados especialmente por el agua continental que recorre la zona costera de origen kárstico propia de la península de Yucatán (Cervantes 2007). Otros procesos involucrados son la captación de $\mathrm{CO}_{2}$ por la actividad fotosintética tanto de la vegetación litoral como por la vegetación sumergida incluyendo el fitoplancton (Hernández-Ayón et al. 2003).

Autores como Castillo-Rivera (1995), Castillo-Rivera \& Zárate-Hernández (2001) y Espinosa-Carreón et al. (2001) han considerado que las condiciones ambientales físicas y químicas que se presentan en la zona costera, juegan un papel importante en la determinación de la variación espacio temporal de la abundancia y diversidad de peces. Sin embargo, Arenas \& Salas (2005) han descrito cuatro elementos que consideran determinantes para la interconectividad de los ecosistemas regionales y el mayor o menor éxito de reclutamiento de diversas estrategias de vida; los giros anticiclónicos y ciclónicos procedentes de la Corriente de Lazo, la presencia de un amplio giro ciclónico en la Bahía de Campeche, la variación en la intensidad en el abundante flujo de agua continental y la cada vez mayor presencia de estructuras artificiales dedicadas a la extracción del petróleo.

Con esta amplia visión es que los resultados de la abundancia y diversidad de la comunidad de peces encontrada en la RBLP es comparada con lo reportado para sistemas adyacentes, en virtud de la ausencia de reportes específicos. El primer nivel de comparación, por la similitud del método de muestreo se realiza con lo reportado por Can (2010) que integra a 48 especies de peces de las cuales $30(62.5 \%)$ coinciden con nuestros resultados, destacando a las familias Lutjanidae y Sparidae como las más diversas por presentar más de cuatro géneros. Nuestros resultados destacan a las familias Tetraodontidae, Lutjanidae y Sparidae como las más diversas.

En el caso de sistemas adyacentes a la RBLP, Ayala-Pérez et al. (2003) reportaron para el Sureste del Golfo de México a 107 especies de peces de las cuales 32 (29.9\%) concuerdan con nuestros resultados y con la familia Sparidae al presentarse 3 géneros y 4 especies, lo cual permite identificar la magnitud del uso de hábitat de estas especies que vinculan a la Laguna de Términos y el litoral del Golfo de México.

Vega-Cendejas (2004) reporta que las familias Sciaenidae, Sparidae y Carangidae comprenden al mayor número de individuos en la Reserva de la Biósfera Ría Celestún, mientras que para la RBLP las familias Sparidae, Haemulidae y Gerreidae integran la mayor abundancia numérica.

Un caso particular reportado por TorresCastro et al. (2008) quienes estudiaron la ictiofauna de petenes y ojos de agua temporales en la porción continental de la RBLP, permite discutir la estrecha vinculación con la región marina adyacente ya que al comparar las 44 especies encontradas, dos de ellas (Eucinostomus argenteus y Cichlasoma urophthalma) son coincidentes con nuestros resultados.

La variación espacial de la abundancia (densidad y biomasa) se asocia directamente a la distribución de los parches de vegetación sumergida. Dichos parches están principalmente constituidos por pastos de Thalassia testudinum, sin embargo, se reconocen también otras especies de fanerógamas y macroalgas. Sin duda y de acuerdo a lo reportado por otros autores como Raz-Guzmán (1995) y AyalaPérez et al. (2008), la vegetación sumergida es un hábitat preferido para múltiples especies de peces ya que además de abrigo ofrece alimento. Se identifica que los sitios de recolecta más cercanos a la línea de costa, muestran mayores pulsos de abundancia lo cual se asocia con la utilización de las raíces de manglar de borde como un hábitat de alimentación y refugio.

En el caso de la variación espacial de la diversidad es posible reconocer los efectos de la dominancia de L. rhomboides, especialmente en el sitio de recolecta 13, que se localiza cercano a la Isla Jaina y cuenta con abundante vegetación sumergida, y está bordeado de manglar, que sin duda ofrece las mejores condiciones para el desarrollo de la especie. El 
contraste de los valores de diversidad con los reportados para otras regiones adyacentes a la RBLP debe ser realizado con cautela debido a que se debe considerar la diversidad de hábitat que se integra al área de estudio respectiva. En este sentido la región de la Laguna de Términos es más diversa que la RBLP sin embargo, como ecosistema lagunar estuarino en la Laguna de Términos la comunidad nectónica cuenta con más alternativas para desarrollar una mayor cantidad de nichos (Ramos-Miranda et al. 2005).

En la escala temporal la variación de la abundancia y en particular del peso promedio se identifican tendencias que pueden ser discutidas en función de la temporalidad climática de la región ya que durante la época de lluvias hay una tendencia de incremento hasta agosto cuando el pulso es máximo, posteriormente durante la época de Secas los valores se mantienen relativamente altos para descender en Octubre, y se identifican como momentos de reclutamiento por el incremento en la densidad pero la disminución en la biomasa y peso promedio. Es necesario profundizar en el análisis de frecuencia de tallas de algunos grupos de especies de manera que se pueda sustentar este planteamiento, sin embargo existe literatura que hace evidente esta programación estacional que han desarrollado los peces costeros como lo describe Lara-Domínguez \& Yáñez-Arancibia (1999), Ayala-Pérez (2006), Ayala-Pérez et al. (2008).

Para el caso de la diversidad, equidad y riqueza temporal, se contrasta con lo reportado por Ayala-Pérez et al. (2003) para la Laguna de Términos; Can (2010) para el litoral frente a la ciudad de Campeche; Arceo-Carranza \& Vega-Cendejas (2009) para la costa central de Yucatán. Aunque estos parámetros son propios de cada sistema ecológico como lo consigna Nagelkerken et al. (2001), el contraste permite identificar la transición entre sistemas que son adyacentes geográficamente pero además valorar la capacidad de algunas especies para interactuar en diferentes comunidades.

De las once especies dominantes destacan L. rhomboides (Familia: Sparidae) y
H. plumierii (Familia: Haemulidae) que en conjunto aportan un $59 \%$ en peso y $67 \%$ en número de individuos del total de la captura. Sosa-López et al. (2010) reportan a estas dos especies como dominantes en el litoral Campechano, juntas representaron el $25 \%$ de la abundancia total. Lo anterior refleja el éxito biológico de estas especies y el rol significativo que representan para la RBLP.

La asociación de especies identificada a partir del dendrograma permite discutir el uso del hábitat a partir de las preferencias de las especies. En este sentido A. quadricornis y C. penna, que se integran en el primer grupo, constituyen especies eurihalinas que prefieren zonas donde la vegetación sumergida es abundante. Ambas se consideran recursos pesqueros (Mexicano-Cíntora et al. 2007), sin embargo las tallas de los organismos recolectados no superan los $20 \mathrm{~cm}$.

El segundo grupo incluye a $S$. foetens, $S$. hispidus y $N$. usta, que en general se observan como especies estenohalinas más asociadas a fondos rocosos y arenosos relativamente someros e incluso arrecifales con presencia de algas del género Sargassum (Bedia \& Franco 2008, Casas-Valdez et al. 2006, Hoffmayer et al. 2005). Fueron más abundantes en la porción Norte del área de estudio, en los sitios 1, 2 y 3 los cuales tienen una comunicación con un canal que va hacia un Peten. Particularmente, $N$. usta mostró un pulso de abundancia en el sitio 12 que se encuentra alejado de línea de costa, lo que sustenta su preferencia por ambientes marinos.

El tercer grupo conformado por $H$. plumierii, O. chrysoptera, E. gula y L. rhomboides, integra a las especies más abundantes entre las dominantes. Estas especies son generalistas que se alimentan de pequeños peces, crustáceos, poliquetos y nemátodos que habitan sobre praderas de pastos marinos (Morales-López et al. 2007), lo que explica su amplia distribución espacial y temporal.

Finalmente el grupo integrado por $A$. rhomboidalis y $O$. beta representa a las especies que se encuentran frecuentemente en fondos lodosos, entre raíces de manglar y sobre fondos 
arenosos cubiertos por vegetación sumergida. A pesar de que muestran preferencias alimenticias contrastantes ya que $A$. rhomboidalis, a pesar de sus marcadas variaciones alimentarias de acuerdo a su crecimiento, en estado adulto se le identifica como herbívora (Guevara et al. 2007), y $O$. beta es un carnívoro voraz, que incluye en su dieta peces pequeños como góbidos, crustáceos (camarón y cangrejos), anélidos y moluscos (Bester 2012). Su asociación se sustenta por la preferencia de uso de hábitat de pastos marinos donde la primera ramonea sobre las hojas de T. testudinum y la segunda aprovecha la disponibilidad de especies de tallas pequeñas que utilizan el área para crianza y protección (Ortiz 2005).

El análisis de correspondencias canónicas permite identificar una relación estrecha entre alta salinidad y la abundancia de $H$. plumierii, lo cual concuerda con la capacidad estenohalina de la especie que también ha sido descrita por Marshall \& Elliot (1998).

Contrariamente $N$. usta, E. gula y $O$. chrysoptera poseen una capacidad euritermohalina lo que les permite ingresos temporales a ambientes salobres e incluso dulceacuícolas (Mexicano-Cíntora, 1999). Arceo-Carranza \& Vega-Cendejas (2009) mencionan que es poco probable que la tolerancia térmica por si misma sea el principal factor para influir en la distribución de los peces, sino que ésta distribución también se debe a una sinergia entre los factores bióticos y abióticos, influyendo en la composición de los ensambles de peces: la preferencia de hábitat, la relación predador-presa, la disponibilidad de alimento y a menudo la biología reproductiva de las especies. Finalmente, algunas especies dominantes como $L$. rhomboides y $O$. beta no mostraron ninguna relación significativa con las variables ambientales, de manera que su distribución espacial es más homogénea.

La macrofauna acuática más diversa y abundante en la RBLP la constituyen los peces y el análisis de su estructura, diversidad y abundancia permite entender procesos de acoplamiento de los diversos ciclos de vida de las especies con la variabilidad ambiental espacial y temporal. Las especies de peces tienen una importancia tanto económica como ecológica ya que son utilizados como recursos pesqueros pero además, funcionan como medios de transporte y transformación de energía, que sin duda colabora sustancialmente al funcionamiento del ecosistema.

De acuerdo con Holmlund \& Hammer (1999) las poblaciones de peces generan diversos servicios a los ecosistemas destacando de manera particular la aportación a los procesos de resiliencia, de tal forma que como área natural protegida la información que se aporta da soporte a estrategias de toma de decisión sobre todo en el caso de aprovechamiento de recursos pesqueros y de recursos ecoturísticos.

\section{AGRADECIMIENTOS}

A la Universidad Autónoma Metropolitana Unidad Xochimilco; al Consejo Nacional de Ciencia y Tecnología y al Instituto de Ecología Pesquerías y Oceanografía del Golfo de México de la Universidad Autónoma de Campeche.

\section{RESUMEN}

La Reserva de la Biósfera Los Petenes constituye un hábitat crítico para una gran diversidad de especies de peces, la zona de pastos marinos es utilizada con fines de alimentación, protección, crianza y refugio temporal. El objetivo es analizar la estructura espacio-temporal de la comunidad de peces y su relación con la variabilidad ambiental e identificar las especies dominantes. Se realizaron 12 muestreos mensuales en 24 sitios a partir de mayo 2009 hasta abril 2010. Se realizaron 288 arrastres, se capturó un total de 21795 organismos con un peso total de $279.5 \mathrm{~kg}$. Se identificaron 46 especies de peces (34 géneros y 23 familias). Los intervalos de variación espacial de la abundancia fueron: $0.018-0.094 \mathrm{ind} . / \mathrm{m}^{2} ; 0.249-1.072 \mathrm{~g} /$ $\mathrm{m}^{2}$ y 9.75-19.32g/ind. Los índices de diversidad fueron: H'n=1.46-2.15bits/ind., J'=0.45-0.71 y D'=2.08-3.92. La variación temporal de la abundancia y diversidad fue de $0.026-0.066$ ind. $/ \mathrm{m}^{2} ; 0.342-0.764 \mathrm{~g} / \mathrm{m}^{2}$ y $6.49-22.98 \mathrm{~g} / \mathrm{ind}$. $\mathrm{H}^{\prime} \mathrm{n}=1.76-2.08 ; \mathrm{J}=0.52-0.64$ y $\mathrm{D}=3.07-4.18$. Se identificaron 11 especies dominantes, que representan el $94.39 \%$ en número de individuos y $89.66 \%$ en peso de la captura total. El análisis cluster permite identificar cuatro grupos de especies que se asocian a las distintas condiciones de hábitat de la reserva. El análisis de correspondencia canónica destaca la asociación de $H$. plumierii con la salinidad y los sólidos disueltos. La RBLP cuenta con una alta diversidad 
de hábitats y la comunidad de peces ha desarrollado estrategias para aprovechar todas las condiciones espaciales y temporales y así satisfacer sus necesidades en el desarrollo de sus ciclos de vida.

Palabras clave: abundancia, diversidad, comunidad de peces, Los Petenes, Campeche.

\section{REFERENCIAS}

Arceo-Carranza, D. \& M.E. Vega-Cendejas. 2009. Spatial and temporal characterization of fish assemblages in a tropical coastal system influenced by freshwater inputs: northwestern Yucatan peninsula. Rev. Biol. Trop. 57: 89-103.

Arenas, V. \& J. Salas. 2005. El Golfo de México. Una aproximación a los determinantes de su biodiversidad, p. 7-22. In J. Hernández Aguilera, J.A. Ruiz Nuño, R. Toral Almazán \& V. Arenas (eds.). Camarones, langostas y cangrejos de la costa este de México, vol. 1. Conabio-Estudio y Conservación de la Naturaleza, A.C., México.

Ayala-Pérez, L.A. 2006. Modelo de simulación de la comunidad de peces en el Área Natural Protegida Laguna de Términos, Campeche, México. Tesis de Doctorado, Universidad Autónoma Metropolitana, México.

Ayala-Pérez, L.A., J. Ramos-Miranda \& D. Flores-Hernández. 2003. La comunidad de peces de la Laguna de Términos: estructura actual comparada. Rev. Biol. Trop. 51: 783-794.

Ayala-Pérez, L.A., J. Ramos-Miranda, O. Chávez-Rivero, G.L. Flores-Beltrán \& V. Nava-Hernández. 2008. La comunidad de peces asociada a la pesca del camarón en la porción occidental de la costa de Campeche, México. Actas de la XVII Bienal de la Real Sociedad Española de Historia Natural. Madrid.

Bautista, F., E. Batllori-Sampedro, G. Palacio, M. OrtizPérez \& M. Castillo-González. 2005. Integración del conocimiento actual sobre los paisajes geomorfológicos de la Península de Yucatán, p. 33-58. In F. Bautista \& G. Palacio. Caracterización y Manejo de los suelos de la Península de Yucatán: Implicaciones Agropecuarias, Forestales y Ambientales. Universidad Autónoma de Campeche, Universidad Autónoma de Yucatán, Instituto Nacional de Ecología.

Bedia, C.S. \& L.J. Franco. 2008. Peces de los sistemas costeros del Estado de Veracruz. Facultad de Estudios Superiores Iztacala, Universidad Nacional Autónoma de México, México.

Braak, C.J.F. ter \& P. Šmilauer. 2002. Canoco for Windows. Version 4.5. Biometrics, Plant Research International, Wageningen.

Can, G.M. 2010. Diversidad y abundancia de peces en la porción litoral frente a la ciudad de San Francisco de Campeche (Febrero 2008-enero 2009). Tesis de Licenciatura, Universidad Autónoma de Campeche, México.

Casas-Valdez, M., H. Marín-Álvarez, R.N. Águila-Ramírez, C.J. Hernández-Guerrero, I. Sánchez-Rodríguez \& S. Carrillo-Domínguez. 2006. El alga marina Sargassum (Sargassaceae): una alternativa tropical para la alimentación de ganado caprino. Rev. Biol. Trop. 54: 83-92.

Castillo-Rivera, M. \& R. Zárate-Hernández. 2001. Patrones espacio-temporales de la abundancia de peces en la laguna de Pueblo Viejo, Veracruz. Hidrobiológica 11: 75-84.

Castillo-Rivera, M. 1995. Aspectos ecológicos de la ictiofauna de la laguna de Pueblo Viejo, Veracruz. Tesis de Maestría, Universidad Nacional Autónoma de México, México. Castro-Aguirre, J.L., P.H. Espinoza \& J.J. Schmitter-Soto. 1999. Ictiofauna estuarinolagunar y vicaria de México. Editorial Limusa, México D.F., México.

Cervantes, M.A. 2007. El balance hídrico en cuerpos de agua cársticos de la Península de Yucatán. Universidad de Quintana Roo, México. Teoría y Praxis 3: 143-152.

Cervigón, F., R. Cipriani, W. Fischer, L. Garibaldi, M. Hendrickx, A.J. Lemus, R. Marquez, J.M. Poutiers, G. Robaina \& B. Rodríguez. 1992. Guía de campo de las especies comerciales marinas de aguas salobres de la costa septentrional del sur de América. FAO, Roma.

CONANP (Comisión Nacional de Áreas Naturales Protegidas). 2006. Programa de conservación y manejo Reserva de la Biósfera Los Petenes. Dirección General de Manejo para la Conservación. Comisión Nacional de Áreas Naturales Protegidas, México D.F., México.

Corbalá, J.A., J. Del Río \& M.J.D. García. 2007. Diversidad, distribución y abundancia de moluscos en la región de Los Petenes. Escuela Superior de Ciencias Agropecuarias. Universidad Autónoma de Campeche. Bol. Inf. JAINA en línea 17: 1-15.

Durán, G.R. 1995. Diversidad florística de los Petenes de Campeche, México. Acta Botánica Mexicana 31: 73-84.

Espinosa-Carreón, T.L., G. Gaxiola-Castro, J.M. RoblesPacheco \& S. Nájera-Martínez. 2001. Temperatura, salinidad, nutrientes y clorofila en aguas costeras de la ensenada del sur de California. Ciencias Marinas 27: 397-422.

Fischer, W. 1978. FAO Species identification sheets for fishery purposes. Western Central Atlantic. (Fishing area 31). FAO, Roma.

Guevara, E., A.J. Sánchez, C. Rosas, M. Mascaró \& R. Brito. 2007. Asociación trófica de peces distribuidos en vegetación acuática sumergida en la Laguna de 
Términos, Sur del Golfo de México. Universidad y Ciencia 23: 151-166.

Hernández-Ayón, J., A. Zirino, S.G. Marinone, R. CaninoHerrera \& M.S. Galindo-Bect. 2003. Relación pHdensidad en el agua de mar. Ciencias Marinas 29: 497-508.

Hoffmayer, E.R., J.S. Franks, B.H. Comyns, J.R. Hendon \& R.S. Waller. 2005. Larval and Juvenile Fishes Associated with pelagic Sargassum in the Northcentral Gulf of Mexico. 56 $6^{\text {th }}$ Gulf and Caribbean Fisheries Institute 56: 259-270.

Holmlund, C.M. \& M. Hammer. 1999. Ecosystem services generated by fish populations. Ecol. Econ. 29: 253-268.

Koranteng, K.A. 2001. Structure and dynamics of a demersal assemblage on the continental shelf an upper slope off Ghana, West Africa. Mar. Ecol. Prog. Ser. 220: 1-12.

Lara-Domínguez, A.L. \& A. Yáñez-Arancibia. 1999. Productividad secundaria, utilización del hábitat y estructura trófica, p. 153-166. In A. Yáñez-Arancibia \& A.L. Lara-Domínguez (eds.). Ecosistemas de Manglar en América Tropical. Instituto de Ecología A.C. México, UICN/ORMA, Costa Rica, NOAA/NMFS Silver Spring, Maryland, USA.

Lara-Lara, J.R., V. Arenas-Fuentes, C. Bazán-Guzmán, V. Díaz-Castañeda, E. Escobar-Briones, M.C. GarcíaAbad, G. Gaxiola-Castro, G. Robles-Jarero, R. SosaÁvalos, L.A. Soto-González, M. Tapia-García \& J.E. Valdez-Holguín. 2008. Los ecosistemas marinos. En: Capital natural de México, vol. I: Conocimiento actual de la biodiversidad. CONABIO, México.

Lohrenz, S.A., D.A. Wiesenburg, R.A. Arnone \& X. Chen. 1999. What controls primary production in the Gulf of Mexico?, p. 151-170. In H. Kumpf, K. Steidinger $\&$ K. Sherman (eds.). The Gulf of Mexico large marine ecosystem: Assessment, sustainability, and management. Blackwell Science, Massachusetts, USA.

Margalef, R. 1969. Perspectives in ecological theory. The University of Chicago, Chicago, USA.

Marshall, S. \& M. Elliot. 1998. Environmental influences on the fish assemblage of the Humber estuary, U.K. Estuar. Coast. Shelf Sci. 46: 175-184.

Mas, J.F. \& J. Correa. 2000. Análisis de la fragmentación del paisaje en el área protegida "Los Petenes", Campeche, México. Investigaciones Geográficas, Bol. Inst. Geogr. UNAM. 43: 43-59.

Méndez-Cabrera, F. \& S. Montiel. 2007. Diagnóstico preliminar de la fauna y flora silvestre utilizada por la población maya de dos comunidades costeras de Campeche, México. Universidad y ciencia 23: $12-139$

Mexicano-Cíntora, G. 1999. Crecimiento y reproducción de la mojarra Eucinostomus gula en Celestún, Yucatán,
México. Proceedings of the Gulf and Caribbean Fisheries Institute 45: 524-536.

Mexicano-Cíntora, G., C.O. Leonce-Valencia, S. Salas \& M.E. Vega-Cendejas. 2007. Recursos pesqueros de Yucatán: fichas técnicas y referencias bibliográficas. CINVESTAV-IPN Unidad Mérida, Mérida, Yucatán, México.

Morales-López, N., E. Pérez-Díaz \& T. Brulé. 2007. Análisis espacio temporal de los ensamblajes de peces presentes en áreas de pastos marinos en la laguna Yalahau, Quintana Roo. México. 59 ${ }^{\text {th }}$ Gulf and Caribbean Fisheries Institute. GCFI 59: 327-334.

Nagelkerken, I., S. Kleijnem, T. Klop, R.A. Van den Brand, E. Cocheret de la Moriniére \& G. Van Der Velde. 2001. Dependence of Caribbean reef fishes on mangroves and seagrass beds as nursery habitats: a comparison of fish faunas between bays with and without mangroves/seagrass beds. Mar. Ecol. Prog. Ser. 214: 225-235.

Ortiz, B.G. 2005. Estructura de la comunidad de peces en un ambiente con vegetación sumergida de una laguna costera tropical. Tesis de Maestría, Universidad Autónoma Metropolitana, México.

Pielou, E.C. 1996. The measurement of diversity in different types of biological collections. J. Theoret. Biol. 13: 131-144.

Ramos-Miranda, J., D. Mouillot, D. Flores-Hernández, A. Sosa-López, T. Do-Chi \& L.A. Ayala-Pérez. 2005. Changes in four complementary facets of fish diversity in a tropical coastal lagoon after 18 years: a functional interpretation. Mar. Ecol. Prog. Ser. 304: 1-13.

Ramos-Miranda, J., K. Berajarano-Hau, D. Flores-Hernández \& L.A. Ayala-Pérez. 2009. Growth, mortality, maturity, and recruitment of the star drum (Stellifer lanceolatus) in the southern Gulf of Mexico. Ciencias Marinas 35: 245-257.

Raz-Guzmán, A. 1995. Caracterización trófica de los componentes dominantes de las comunidades bentónicas en Laguna de Términos, Campeche y en la plataforma continental adyacente. Tesis de Doctorado, Instituto de Ciencias del Mar y Limnología, UNAM, México D.F., México.

Rico-Gray, V. 1982. Estudio de la vegetación de la zona costera inundable del noroeste del Estado de Campeche, México, Los Petenes. Biótica 7: 171-190.

Shanon, C.E. \& W. Weaver. 1963. The mathematical theory of communication. University of Illinois, Urbana, USA.

Sosa-López, A., J. Ramos-Miranda, D. Flores-Hernández, F. Gómez-Criollo \& L.A. Ayala-Pérez. 2010. El Chac-Chi y la Xlavita en la Reserva de la Biósfera "Los Petenes". Explora 2: 8-11. 
Suárez-Morales, E. \& E. Rivera-Arriaga. 1998. Hidrología y fauna acuática de los cenotes de la península de Yucatán. Rev. Soc. Mex. Hist. Nat. 48: 37-47.

Systat Software Inc. 2002. Chicago, USA. www.systat.com

Torres-Castro, I.L., M.E. Vega-Cendejas, J.J. SchmitterSoto, G. Palacio-Aponte \& R. Rodiles-Hernández. 2008. Ictiofauna de sistemas cárstico-palustres con impacto antrópico: Los Petenes de Campeche, México. Rev. Biol. Trop. 57: 141-157.

Vega-Cendejas, A.E. 2004. Ictiofauna de la Reserva de la Biosfera Celestún, Yucatán: una contribución al conocimiento de su biodiversidad. Anales del Instituto de Biología. Serie Zoología 75: 193-206.

Vidal, V.M., F.V. Vidal, A.F. Hernández, E. Meza \& J.M. Pérez Molero. 1994. Baroclinic flows, transports, and kinematic properties in a cyclonic-anticycloniccyclonic ring triad in the Gulf of Mexico. J. Geophys. Res. 99: 7571-7597.
Villalobos-Zapata, G. 2004. Reservas de la Biósfera: Los Petenes y Ría Celestún, p. 397-412. In RiveraArriaga, E., G.J. Villalobos-Zapata, I. Azuz-Adeath \& F. Rosado-May (eds.). El Manejo Costero en México. UAC/CETYS Univesidad/UQROO/SEMARNAT. México.

Vukovich, F.M. 1988. Loop current boudary variations. J. Geophys. Res 93: 15585-15591.

Wiseman, W.J. \& W. Sturges. 1999. Physical oceanography of the Gulf of Mexico: Processes that regulate its biology, p. 77-91. In H. Kumpf, K. Steidinger \& K. Sherman. The Gulf of Mexico large marine ecosystem: Assessment, sustainability, and management. Blackwell Science, Massachusetts, USA.

Yáñez-Arancibia, A. \& J.W. Day Jr. 1982. Ecological characterization of Terminos Lagoon, a tropical lagoon-estuarine system in the Gulf of Mexico ISCOL-UNESCO, Bordeaux France Sept. 7-14. Oceanologica Acta. 
